\section{Juvenile ossifying fibroma of maxilla}

Received: 30 December 2009 / Accepted: 2 March 2010

(C) Association of Oral and Maxillofacial Surgeons of India 2009

\section{Yadavalli Guruprasad $^{1} \otimes \cdot$ Girish Giraddi $^{2}$}

${ }^{1}$ Asst. Professor and Consultant, Dept. of Oral and Maxillofacial Surgery, AME'S Dental College Hospital \& Research Centre, Raichur

${ }^{2}$ Professor and Head, Dept. of Oral and Maxillofacial Surgery, Govt. Dental College \& Research Institute, Bangalore

\section{Address for correspondence:}

\section{Yadavalli Guruprasad}

Assistant Professor \& Consultant

Maxillofacial Surgeon

Dept. of Oral and Maxillofacial Surgery

AME'S Dental College Hospital and

Research Centre, Raichur-584103

Karnataka, India

$\mathrm{Ph}:+919886690065$

E-mail: guru_omfs@yahoo.com

Keywords Juvenile ossifying fibroma $\cdot$ Maxilla $•$ Fibroosseous lesions

\section{Introduction}

Fibroosseous lesions are a diverse group of processes that are characterized by replacement of normal bone by fibrous tissue containing a newly formed mineralized product. Commonly included among the fibroosseous lesions of the jaws are fibrous dysplasia, cemento-osseous dysplasia and ossifying fibroma [1].

Juvenile Ossifying Fibroma (JOF) is an uncommon, benign, bone-forming neoplasm that is distinguished from other fibro-osseous lesions primarily by its age of onset, clinical presentation, and potential behavior. This lesion most often occurs between 5 and 15 years of age $[1,2]$ shows a male predilection [2-5], and may exhibit rapid growth of the involved anatomic site [6], sometimes resulting in considerable facial disfigurement. Most juvenile ossifying fibromas arise in the vicinity of the paranasal sinuses [5]. With regard to the incidence of JOF in the jaws, there are conflicting reports of maxillary $[2,5,7]$, and mandibular $[3,4]$ predilections. A recent study by El-Mofty identified two histopathological variants, trabecular JOF (TrJOF) and psamamatoid (PsJOF). One clinical feature that helps differentiate TrJOF from PsJOF is the site of involvement, with PsJOF occurring mainly in the paranasal sinuses and TrJOF occurring mainly in the maxilla [4].
The aggressive nature of this entity, along with the reported high rates of recurrence (30-58\%), suggests that JOF should be treated like a locally aggressive neoplasm. Surgical resection rather than conservative curettage is therefore the preferred line of treatment [4]. This report details the diagnosis and treatment of a 14year-old girl presenting with a maxillary swelling that was subsequently determined to be JOF.

\section{Case report}

A 14-year-old girl was referred to the Department of Oral and Maxillofacial Surgery at Govt. Dental College and Research Institute, Bangalore for the evaluation of a left facial swelling (Fig. 1). Both the patient and her parents had been aware of the swelling for approximately 2 years, but delayed evaluation because of economic circumstances. The lesion had been slowly increasing in size since it was first noticed. There was no history of trauma, pain, paresis, paresthesia or lymphadenopathy. Physical examination showed a healthy, normally developed young girl in no apparent distress. There was significant facial asymmetry caused by an approximately $5 \times 5 \mathrm{~cm}$ mass involving the left maxilla. The mass was firm and nontender to palpation and not adherent to the overlying skin. No bruits or pulsations were detected. Intra-oral examination showed expansion of the left buccal cortex, which was firm on palpation. There was no evidence of tooth mobility, abscess formation, dehiscence or malocclusion. A panoramic radiograph showed a wellcircumscribed radiopacity with faint, linear to irregular central opacities. There was evidence of tooth displacement in relation to $21,27,28$ and root resorption in relation to 21, 26 (Fig. 3). Teeth 23, 24 and 25 were noted to be missing with history of extraction due to mobility (Fig. 2). Computed Tomography (CT) scan of the left maxilla showed medial and lateral cortical expansion and areas of central calcification with obliteration of the left maxillary sinus (Fig. 4). Laboratory values were within normal limits. An incisional biopsy was subsequently performed, providing a diagnosis of juvenile ossifying fibroma.

Approximately two weeks later, the lesion was excised with the patient under general anesthesia. As the mass was extensive in size it was approached using Weber-Fergusson incision with a lateral extension (Fig. 5). Inspection of the bony cavity found no cortical dehiscence. A peripheral ostectomy of the bony cavity then was performed. Grossly, the specimen measured approximately $4.5 \times 3.5 \times 1.5 \mathrm{~cm}$ and had a smooth, lobulated outer surface with a pale, firm, homogenous cut surface and was well encapsulated (Fig. 6). 


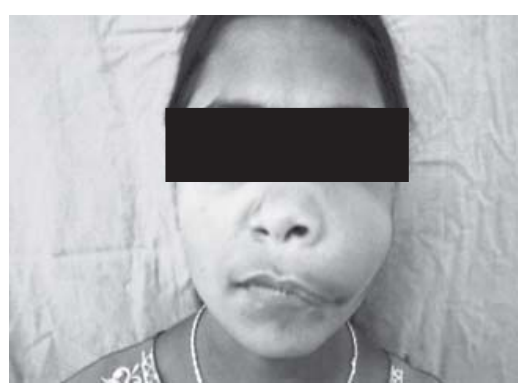

Fig. 1 Clinical appearance of the lesion at the time of evaluation

Microscopic examination showed a cellular connective tissue stroma consisting of fibroblasts arranged in a whorled or storiform pattern, which merged into anastomosing areas of cellular condensation. There were ribbon like osteoid trabeculae lined by numerous plump osteoblasts that showed a transition into areas of woven bone associated with occasional osteoclasts. Clusters of multinucleated giant cells were noted near areas of cystic change and hemorrhage. In some regions, round to oval calcified spherules were imbedded in the stroma and was encapsulated (Fig. 7). Based on the clinical history, radiographic and histologic features of the lesion, a diagnosis of juvenile ossifying fibroma of trabecular type was established. The patient was discharged from the hospital on second postoperative day and sutures were removed after one week; removable prosthesis was given after six months (Figs. $8,9)$ and followed up for one year.

\section{Discussion}

JOF may present clinically as either a gradual or rapid, painless expansion of the affected bone or region. The main characteristics are: a patient under 15 years of age, the location of the tumour, the radiologic pattern, and tendency to recur. JOF is often seen in a very young child. In reviews published by Hamner et al. and Slootweg et al. the mean age of onset was 11.5 and 11.8 years old, respectively [9].

Clinically, this lesion has in general a more aggressive growth rate than ossifying fibroma. Most cases of maxillary JOF are asymptomatic, as was the present case.

Nasal obstruction, exophthalmos and, rarely, intracranial extension can be associated with those lesions arising within the paranasal sinuses and orbit $[5,6]$. Radiographic features are non-specific and depending on the location of the tumour, maturation stage and stage of ossification,

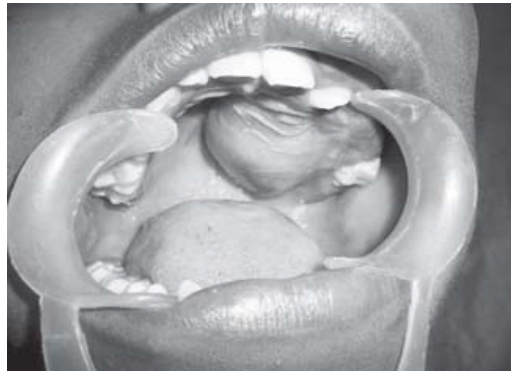

Fig. 2 Intra-oral swelling in the region of the palate, labial \& buccal sulcus region

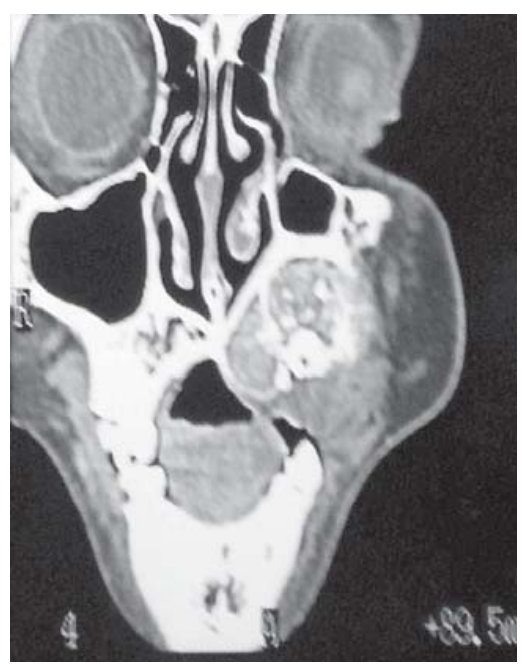

Fig. 4 Coronal CT showing obliteration of left maxillary sinus \& displacement of teeth

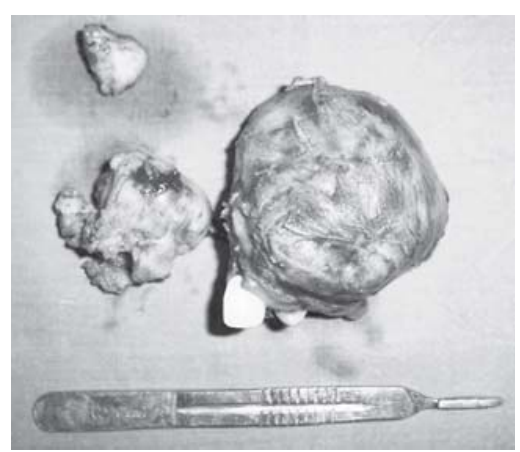

Fig. 6 Photograph of the excised tumour

they are uni or multilocular well-defined lesions which may be radiolucent, mixed or radioopaque [6]. Aggressive lesions may show cortical thinning and perforation [8]. The most frequent location of JOF is in the paranasal sinuses, accounting for about $90 \%$ of cases, where as mandibular lesions account for approximately $10 \%$ of the facial JOF cases. In general, JOF has a more aggressive growth pattern than the adult

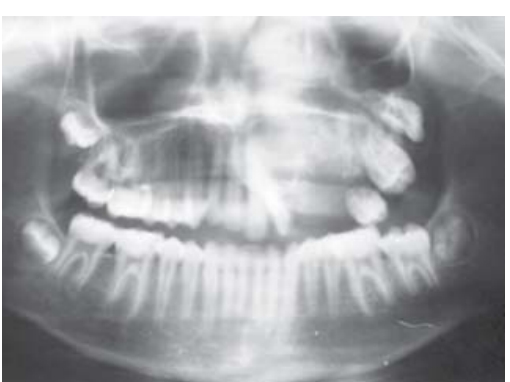

Fig. 3 Panoramic radiograph of the patient showing radiopacity $\&$ displacement of teeth

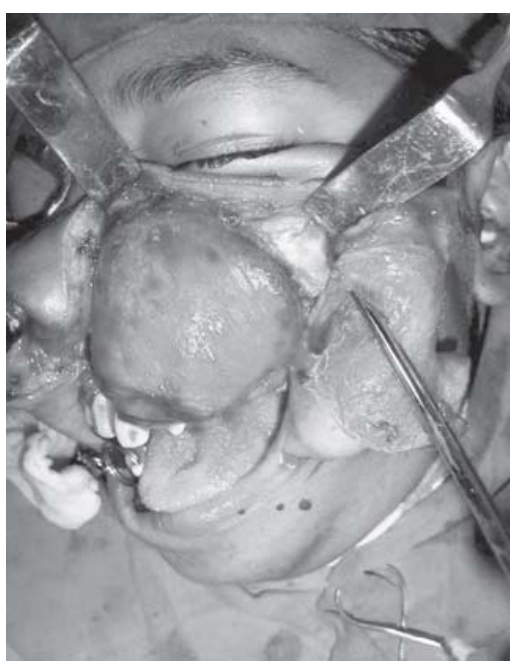

Fig. 5 Intra-operative view of the exposed lesion. Note the smooth surface of the tumour, which allowed it to be easily separated from the surrounding bone

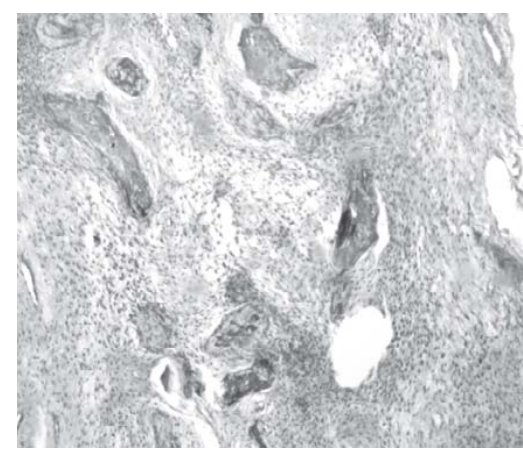

Fig. 7 Microscopically, the tumour showed focal hypercellular regions with anastomosing osteoid trabeculae lined by osteoblasts

variant of ossifying fibromas. They are usually asymptomatic, exhibit rapid growth of the involved site and the first presentation will be a clinically obvious swelling. All the above features were seen in our case.

Rapidly expanding central jaw lesions of children and adolescents, when coupled with the above radiographic features, are worrisome for malignancy. Malignant 


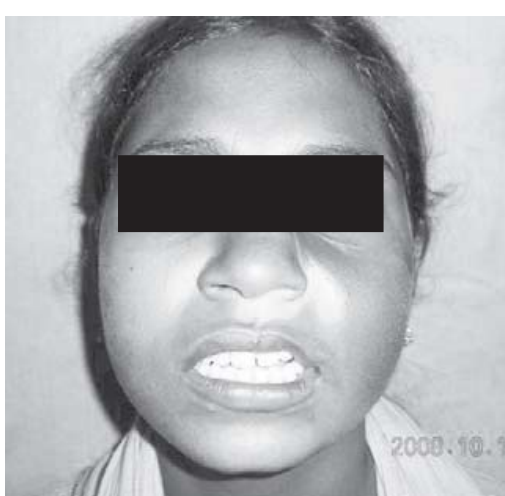

Fig. 8 Postoperative extra-oral photograph after six months

neoplasms that have a tendency to involve the craniofacial skeleton in this age group include Osteosarcoma, Chondrosarcoma, Ewing's sarcoma, and the African form of Burkitt's lymphoma. Benign neoplastic or developmental lesions are more commonly encountered in the jaws, and, although they may show differing degrees of aggressiveness, they often present with the radiographic characteristics of JOF. Included among these diseases are osteoblastoma, an intraosseous form of fibromatosis known as desmoplastic fibroma, cemento-ossifying fibroma, fibrous dysplasia, central giant cell granuloma, and cherubism. There is some degree of histological overlap between JOF and cemento-ossifying fibroma. In contrast to cemento-ossifying fibroma, which histologically is characterized by uniformity of pattern, JOF is more likely to show markedly heterogeneous morphology $[2,6,9]$, a characteristic that may complicate the diagnosis and subsequent management of these tumours. Areas of dense cellularity may alternate with myxomatous regions, and the distribution of bone trabeculae and ossicles often is uneven. Caution must, therefore, be exercised in the interpretation of incisional biopsies of fibro-osseous lesions.

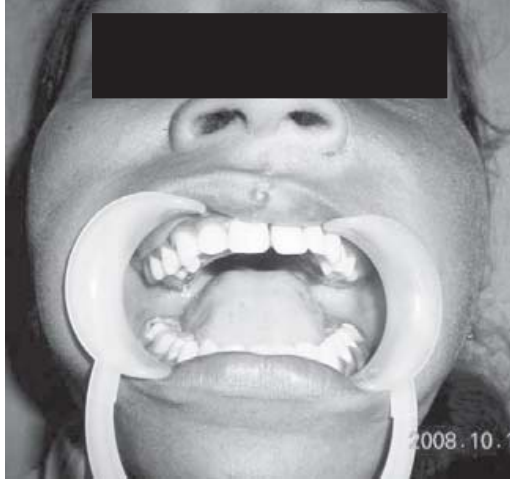

Fig. 9 Postoperative intra-oral photograph after prosthesis

The rapid growth rate often exhibited by these lesions can be quite alarming and cause the clinician to suspect the presence of a malignancy. It is therefore important to maintain active communication between surgeon and pathologist to establish the benign nature of the lesion and prevent overtreatment. Whereas the treatment for JOF recommended by most investigators $[2,6,10,11]$ is conservative excision or curettage, some lesions may necessitate more aggressive management [12]. Longstanding lesions may show significant cortical destruction and periosteal elevation [8], which can increase the risk of recurrence. The recurrence rate ranges from $30 \%$ observed by Johnson et al. to $58 \%$ reported by Makek $[5,6]$ continued followup is essential. Despite the aggressive nature of the lesion and high rate of recurrence, malignant transformation to sarcoma has not been reported.

\section{References}

1. Sun G, Chen X, Tang E, Li Z, Li J (2007) Juvenile ossifying fibroma of maxilla. Int J Oral Maxillofac Surg 36(1): 82-85

2. Slootweg PJ, Muller H (1990) Juvenile ossifying fibroma: Report of four cases.
J Craniomaxillofac Surg 18(3): 125129

3. Williams HK, Mangham C, Speight PM (2000) Juvenile ossifying fibroma. An analysis of eight cases and a comparison with other fibro-osseous lesions. J Oral Pathol Med 29(1): 1318

4. El Mofty S (2002) Psammomatoid and trabecular juvenile ossifying fibroma of the craniofacial skeleton: two distinct clinicopathologic entities. Oral Surg Oral Med Oral Pathol Oral Radiol Endod 93(3): 296-304

5. Johnson LC, Yousefi M, Vinh TN, Heffner DK, Hyams VJ, Hartman KS. (1991) Juvenile active ossifying fibroma: Its nature, dynamics and origin. Acta Otolaryngol Suppl 488: 140

6. Waldron CA (1993) Fibro-osseous lesions of the jaws. J Oral Maxillofac Surg 51(8): 828-835

7. Waldron CA (1985) Fibro-osseous lesions of the jaws. J Oral Maxillofac Surg 43(4): 249-262

8. Noffke CE (1998) Juvenile ossifying fibroma of the mandible. An 8 year radiologic follow-up. Dentomaxillofac Radiol 27(6): 363-366

9. Slootweg PJ (1996) Maxillofacial fibro-osseous lesions: Classification and differential diagnosis. Semin Diagn Pathol 13(2): 104-112

10. Wiedenfeld KR, Neville BW, Hutchins AR, Bell RA, Brock TR (1995) Juvenile ossifying fibroma of the maxilla in a 6year-old male: Case report. Pediatr Dent 17(5): 365-367

11. Yang HY, Zheng LW, Luo J, Yin WH, Yang HJ, Zwahlen RA (2009) Psammomatoid juvenile cementoossifying fibroma of the maxilla. J Craniofac Surg 20(4): 1190-1192

12. Shekhar MG, Bokhari K (2009) Juvenile aggressive ossifying fibroma of the maxilla. J Indian Soc Pedod Prev Dent 27(3): 170-174 